\title{
Fatores Associados à Qualidade de Vida de Docentes da Área da Saúde
}

\author{
Factors Related to Healthcare Teachers' \\ Quality of Life
}

\author{
Lyssa Esteves Souza Souto \\ Sarah Martins Souzal \\ Cássio de Almeida Lima ${ }^{I I}$ \\ Mayara Karoline Silva Lacerda \\ Maria Aparecida Vieira \\ Fernanda Marques da Costa ${ }^{I}$ \\ Antônio Prates Caldeira
}

\section{PALAVRAS-CHAVE}

- Docentes;

- Qualidade de Vida;

- Ensino Superior;

- Educação Médica;

- Universidade.

\section{KEYWORDS}

- Faculty;

- Quality of Life;

- Higher Education;

- Medical Education;

- University.
Reencaminhado em: 14/11/2015

Aprovado em: 26/09/2016

O presente estudo teve como objetivo descrever a qualidade de vida e os fatores associados entre os docentes dos cursos de graduação da área da saúde. Trata-se de pesquisa transversal, de abordagem quantitativa. Teve como cenário uma universidade pública, situada em uma cidade de porte médio no Estado de Minas Gerais, Brasil. Os dados foram organizados e analisados no programa estatístico Statistical Package for Social Sciences (SPSS) 18.0. Aplicou-se a estatística descritiva, o T de Student e Anova/post hoc de Bonferroni para investigar associações. Identificou-se qualidade de vida boa nos domínios psicológico, relações sociais e meio ambiente, e regular no domínio físico. Docentes que exercem a carreira há mais de 12 anos, não têm companheiro, não residem com a família, apresentam idade inferior a 43 anos e atuam nos cursos de Biologia e Medicina apresentaram comprometimento da qualidade de vida. Recomendam-se ações efetivas de promoção da saúde, especialmente para os professores com menores escores de qualidade de vida.

The present study aimed to describe quality of life and associated factors among teachers of undergraduate healthcare courses, by means of transversal research with a quantitative approach. The study was developed at a public university located in a medium-sized city in the state of Minas Gerais, Brazil. The data was organized and analyzed using statistic program Statistical Package for Social Sciences (SPSS) 18.0. Descriptive statistic, the T Student and Anova/post hoc-Bonferroni were all performed. The study identified good quality of life in the areas of psychology, social relations and environment, while the physical domain was regular. Teachers of under 43 who had been working for over 12 years, without a partner and who lived without their family and taught on the biology and medicine courses, showed a major tendency toward poor quality of life. Effective initiatives are recommended to promote health, especially among teachers with lower scores in quality of life. 


\section{INTRODUÇÃO}

A temática da qualidade de vida se refere aos padrões que a própria sociedade define e se mobiliza para conquistar, consciente ou inconscientemente, e ao conjunto das políticas públicas e sociais que induzem e norteiam o desenvolvimento humano. Também se relaciona às modificações positivas no modo, nas condições e estilos de vida. Vale ressaltar que parcela significativa da formulação teórica acerca do assunto e das responsabilidades se reserva à área da saúde ${ }^{1 .}$ Trata-se, ainda, de um assunto em consolidação como uma variável importante na prática clínica e na produção de conhecimento nessa área. Sua aplicação pode resultar em mudanças nas práticas de atenção e na efetivação de novos paradigmas do processo saúde-doença pautados na promoção da saúde, cujos aspectos socioeconômicos, culturais e psicológicos devem ser reconhecidos e se vinculam à qualidade de vida, um construto que se caracteriza, dessa forma, como eminentemente interdisciplinar ${ }^{2}$.

Desde a década de 1980, esse tema é pesquisado com enfoques objetivo e subjetivo. O primeiro refere-se ao grau de satisfação das necessidades em função da sua ampliação socioeconômica, e o segundo diz respeito ao bem-estar da pessoa e sua percepção do nível de realização pessoal nos âmbitos individuais e coletivos ${ }^{3}$.

O conceito de qualidade de vida começou a ser utilizado nos Estados Unidos da América logo após a Segunda Guerra Mundial para expor os bens materiais adquiridos no pós-guerra, como, por exemplo, viagens e investimentos. Adiante, o conceito ampliou-se com a intenção de abarcar o grande desenvolvimento econômico de diversas localidades por meio de indicadores econômicos, como o produto interno bruto e a renda per capita. Com o avançar do tempo, passou a incorporar também o conceito social e a mensurar o desenvolvimento por meio de outros indicadores, tais como moradia, saúde e educação ${ }^{4}$.

Atualmente, a expressão qualidade de vida apresenta uma concepção que envolve parâmetros das áreas de saúde, arquitetura, urbanismo, lazer, gastronomia, esportes, educação, meio ambiente, segurança pública e privada, entretenimento, novas tecnologias e tudo o que se relacione com o ser humano, sua cultura e seu meio. Tem-se expandido ao longo do tempo, englobando o desenvolvimento socioeconômico e humano, aspecto objetivo/subjetivo e a percepção individual. A compreensão da qualidade de vida é mutável de pessoa para pessoa e é dinâmica em cada percepção subjetiva do processo de produção, circulação e consumo de bens e riquezas ${ }^{5}$.

A compreensão ampliada de qualidade de vida também abrange diversos cenários, entre os quais a universidade, que possui papel determinante no desenvolvimento de seus futuros profissionais não só por demonstrar resolubilidade em comparação com instituições de outra natureza, mas também por seu papel singular na definição da ética de desenvolvimento nacional e por ser um lócus especial de crítica e transformação social'.

Nesse panorama, o docente assume função de destaque como agente de saúde, uma vez que não apenas coloca em prática a comunicação de seu saber, como também é um agente de transformações que possui singularidades em seus conhecimentos. Sua prática produz efeitos na formação dos novos profissionais, pois esta passa a ser um vetor importante nos processos de subjetivação do sujeito para a formação profissional e pessoal, tornando essa profissão uma atividade enriquecedora, que proporciona diversas habilidades ligadas ao campo da saúde. Para atender às necessidades da população, é fundamental que tanto os docentes quanto os discentes compreendam o significado de qualidade de vida e o valor que tem para os sujeitos com os quais interagem no cotidiano. Isso porque a forma e a quantidade de processamento de suas atividades é que irão proporcionar ou não a qualidade de vida dessas pessoas ${ }^{7,8}$.

A influência do cotidiano na qualidade de vida dos docentes da área da saúde está diretamente relacionada com diversos fatores. Entre eles, destaca-se que, quanto maior o tempo de dedicação dos docentes ao trabalho, menor é sua disponibilidade para as atividades pessoais cotidianas, como os afazeres domésticos, cuidados com a família, a saúde e o lazer. Sucede-se uma sobrecarga de exigências individuais e familiares que, assim, afeta todo o contexto social $^{8,9}$.

Também é pertinente observar que os locais onde os professores universitários exercem sua profissão são ambientes que afetam diretamente suas vidas, pois há inúmeros obstáculos ligados às transformações articuladas à ciência, à educação e ao trabalho. Nesse sentido, os docentes buscam refletir sobre suas práticas diárias no espaço acadêmico e nos serviços de saúde e, diante dos problemas, almejam consolidar propostas de mudanças e formulação de novos planos consistentes ${ }^{10}$. Sabe-se que a má qualidade de vida interfere no desempenho da saúde física e mental do trabalhador, influencia no desenvolvimento do planejamento e de realização das atividades, e pode estar associada a estresse, tensão nervosa, fadiga muscular, lesões por esforço repetitivo e distúrbios osteomusculares relacionados ao trabalho ${ }^{11}$.

Este estudo teve por objetivo descrever a qualidade de vida e os fatores associados entre os docentes dos cursos de graduação da área da saúde. 


\section{MÉTODO}

Trata-se de um estudo descritivo, transversal, de abordagem quantitativa. Teve como cenário o Centro de Ciências Biológicas e da Saúde do campus universitário sede de uma universidade pública, situada em uma cidade de porte médio no Estado de Minas Gerais, Brasil, que oferece os seguintes cursos: Ciências Biológicas, Educação Física, Enfermagem, Medicina e Odontologia.

Como critério de inclusão, os professores deveriam estar inseridos em atividades de ensino na graduação desses cursos. Foram excluídos os que estavam afastados pelos seguintes motivos: cursando pós-graduação stricto sensu ou pós-doutorado, licença-maternidade, férias regulamentares, licença para tratamento de saúde, em processo de aposentadoria, férias prêmio e licença para tratar de interesses particulares.

Havia, no cenário do estudo, 43 docentes do curso de graduação em Ciências Biológicas, 43 de Educação Física, 65 de Enfermagem, 165 de Medicina e 63 de Odontologia, totalizando 379 professores. Utilizou-se a amostragem probabilística estratificada para a definição da amostra, e a seleção dos participantes ocorreu de forma aleatória. Os cálculos utilizados nessa amostra tiveram como base: prevalência conservadora de $50 \%$ para médias positivas de qualidade de vida, população de 379 docentes, margem de erro de 3,5\% e nível de confiança de $95 \%$. O número identificado foi acrescido de $15 \%$ para eventuais perdas. Dessa forma, o número mínimo de professores para o estudo definido pelo cálculo amostral foi de 295 indivíduos, que foram identificados por sorteio aleatório de cada estrato, ou seja, em cada curso.

A coleta de dados foi efetuada de junho a setembro de 2013 e iniciou-se após a definição e consentimento dos envolvidos quanto aos aspectos éticos e logísticos, em salas de aulas, campos de estágios e em consultórios particulares de alguns professores.

A estratégia utilizada para a coleta foi a aplicação de um questionário que abordava questões sociodemográficas e acadêmicas, e do Whoqol-Breve, instrumento criado pelo Grupo de Qualidade de Vida da Organização Mundial da Saúde (OMS), abreviado a partir do Whoqol-100 (W-100), constituído por 26 questões fechadas, sendo duas perguntas gerais de qualidade de vida e outras 24 representantes de cada uma das 24 facetas que constituem o instrumento original. Essas 24 questões são agrupadas em 4 domínios: físico -7 itens, psicológico -6 itens, relações sociais -3 itens e meio ambiente -8 itens. $\mathrm{O}$ instrumento considera as duas últimas semanas vividas pelos respondentes, e as respostas às questões são dadas numa escala com um único intervalo de 0 a 5 . Os escores finais de cada domínio são calculados por uma sintaxe na qual tais escores podem ser medidos em direção positiva numa variação de 0 a $100^{12}$.

Neste estudo, considerou-se a distribuição dos escores de qualidade de vida estabelecidos conforme os domínios analisados: qualidade de vida ruim (21-40); nem ruim nem boa (4160); boa (61-80) e muito boa, quando os escores variavam entre $81-100^{13}$. As questões (1) Como você avalia sua qualidade de vida? (muito ruim; ruim; nem ruim nem boa; boa; muito boa) e (2) Quão satisfeito(a) você está com a sua saúde? (muito insatisfeito; insatisfeito; nem satisfeito nem insatisfeito; satisfeito; muito satisfeito) foram analisadas separadamente porque não estão incluídas nas equações para a sintaxe do Whoqol.

Também foi aplicado um questionário com a finalidade de obter informações complementares sobre os docentes, referentes aos aspectos sociodemográficos e acadêmicos, possibilitando descrever seu perfil e mensurar possíveis associações com qualidade de vida.

As covariáveis foram agrupadas em características sociodemográficas e acadêmicas. Entre as covariáveis, incluíram-se sexo, idade (dicotomizada usando-se a média como ponto de corte), estado civil (categorizado em casado e união estável e sem companheiro, considerando os solteiros e viúvos), cor autodeclarada (categorizada em pardos e outras, incluindo brancos, negros e indígenas). Além de local de nascimento e residência, com quem reside (categorizada em reside com a própria família e outra situação - reside com amigos ou sozinho), renda familiar que foi categorizada pela média, outro vínculo (sim e não), tempo de docência, categorizado com base na média. Investigou-se, ainda, a leitura de livros, excetuando-se os acadêmicos, a existência de computador com acesso à internet no domicílio, se tem a internet como principal fonte de informação, ambas categorizadas em sim e não. Também foi avaliado se o professor, afora as atividades acadêmicas, ocupa-se mais com atividades culturais, incluindo ir a teatro, cinema, parques e lazer com amigos, ou sociofamiliares - geralmente fica em casa, assiste à TV e frequenta eventos familiares.

Após a coleta, os dados foram organizados e analisados com a utilização do programa estatístico Statistical Package for Social Sciences (SPSS) 18.0. Para a análise de dados, aplicou-se a estatística descritiva com o uso de frequências absolutas e relativas e cálculo de médias e desvios padrões. A fim de investigar associações entre variáveis sociodemográficas e acadêmicas e os domínios da qualidade de vida, procedeu-se à checagem da normalidade dos dados por meio do teste de Klomogorov-Smirnov. Em seguida, realizou-se a análise bivariada, empregando-se a comparação de escores médios de qualidade de vida em cada domínio pelo teste T de Student para amostras independentes e Anova/ pós Hoc de Bonferroni 
para variáveis com três ou mais categorias. A finalidade desses testes foi comparar os escores médios em cada domínio em relação às covariáveis. Em todas as análises, foi utilizado o nível de significância de 5\% (p $\leq 0,05)$.

De acordo com as normas da Resolução nº 466/2012, este estudo foi aprovado por Comitê de Ética em Pesquisa, por meio do Parecer Consubstanciado nº 173.395/2012, Certificado de Apresentação para Apreciação Ética (CAAE) 10944812.3.0000.5146. Todos os participantes assinaram o Termo de Consentimento Livre e Esclarecido.

\section{RESULTADOS}

Do total de 295 docentes definidos pelo cálculo amostral, 221 responderam aos questionários, perfazendo uma taxa de resposta de 75\%. Assim, participaram deste estudo 34 (15,4\%) docentes do curso de Biologia; 35 (15,8\%) de Educação Física; 50 $(22,6 \%)$ de Odontologia; 48 (21,7\%) de Medicina e 54 (24,4\%) de Enfermagem.

As características sociodemográficas dos docentes dos cursos de graduação na área da saúde foram as seguintes: maioria do sexo feminino nos cursos de Biologia (67,6\%), Odontologia (56,0\%), Medicina (56,3\%) e Enfermagem (77,8\%). Entretanto, no curso de Educação Física, a maioria dos docentes era do sexo masculino (51,4\%). A faixa etária até os 43 anos foi predominante entre os docentes de todos os cursos em questão - Biologia 58,8\%, Educação Física 60,0\%, Odontologia 52,0\%, Medicina 51,0\% e Enfermagem 72,2\%.

Quanto ao estado civil, a maioria dos docentes de todos os cursos era casada - Biologia 67,6\%, Educação Física 74,3\%, Odontologia 82,0\%, Medicina 70,8\%, Enfermagem 64,8\%. Além disso, os professores afirmaram ser da cor autodeclarada branca (Biologia 55,9\%, Educação Física 57,1\%, Odontologia 62,0\%, Medicina 58,3\%, Enfermagem 51,0\%); residem na cidade de Montes Claros (Biologia 70,6\%, Educação Física $100,0 \%$, Odontologia 96,0\%, Medicina 100,0\%, Enfermagem $98,1 \%$ ) e moram com a família (Biologia 70,6\%, Educação Física 85,7\%, Odontologia 94,0\%, Medicina 85,4\%, Enfermagem $85,2 \%)$

Quanto a ter outro vínculo empregatício, os docentes dos cursos de Educação Física (51,4\%), Odontologia (60,0\%), Medicina $(85,4 \%)$ e Enfermagem $(75,9 \%)$ afirmaram possuir mais de um vínculo. Mas, entre os docentes do curso de Biologia, verificou-se que $32,4 \%$ possuem outro vínculo empregatício. Em relação ao tempo de atuação na docência, a maioria tem até 12 anos - Biologia 58,8\%, Educação Física 62,9\%, Odontologia $62,0 \%$, Enfermagem $72,2 \%$. Porém, no curso de $\mathrm{Me}-$ dicina, observou-se que a maioria possui 13 anos ou mais de docência $(60,4 \%)$.
Ademais, a maioria possui renda bruta mensal de até 11 salários mínimos - Biologia 64,7\%, Educação Física 68,6\%, Odontologia $62,0 \%$ e Enfermagem $66,7 \%$. Por outro lado, no curso de Medicina, verificou-se que a maioria dos docentes possui renda bruta maior que 12 salários mínimos (64,6\%).

Quanto à formação universitária, a maioria possui mestrado - Biologia 79,4\%, Educação Física 54,3\%, Odontologia 70,0\%, Medicina 54,2\% e Enfermagem 59,3\%; lê acima de dois livros ao ano, excetuando a bibliografia acadêmica - Biologia 70,6\%, Educação Física 54,3\%, Odontologia 56,0\%, Medicina $50,2 \%$ e Enfermagem 63,0\%.

Os docentes, em sua maior parte, não eram fumantes Biologia 79,4\%, Educação Física 88,6\%, Odontologia 92,0\%, Medicina 97,9\% e Enfermagem 100,0\% - e dispõem de outras fontes de informações além da internet - Biologia 61,8\%, Educação Física 71,4\%, Odontologia 82,0\%, Medicina 68,8\% e Enfermagem 59,3\%.

Em relação às atividades de lazer, a maioria prefere atividades culturais - Educação Física 74,3\%, Odontologia 62,0\%, Medicina 54,2\% e Enfermagem 63,0\% -, exceto os docentes do curso de Biologia, que preferem as atividades sociais $(55,9 \%)$.

Quanto à qualidade de vida geral, a maioria dos professores $(75,1 \%)$ avaliou a saúde como boa. Em relação a quão satisfeitos os docentes estão com a própria saúde, a maioria $(69,7 \%)$ respondeu que estava satisfeita.

Os resultados obtidos nas respostas do Whoqol foram expressos por médias e desvios padrões dos escores transformados (percentuais), calculados previamente para cada um dos domínios. A maior variação de desvio padrão ocorreu no domínio "relações sociais", e a menor, no domínio "físico", que se mostrou o mais afetado, pois apresentou menores valores médios $(57,9 ; \mathrm{DP} \pm 9,6)$, ao passo que as "relações sociais", com maior valor $(73,7 ; \mathrm{DP} \pm 17,2)$, foi o aspecto mais positivo da qualidade de vida dos docentes. Em relação ao curso de atuação do docente, verificaram-se maiores escores médios entre os docentes do curso de Odontologia nos domínios físico $(60,3$; DP $\pm 10,9)$ e meio ambiente $(73,9 ; \mathrm{DP} \pm 15,7)$. Foram identificados maiores escores nos domínios psicológico $(69,7 ; \mathrm{DP} \pm 9,3)$ e relações sociais (78,8; DP $\pm 5,0)$ no curso de Educação Física (Tabela 1).

A Tabela 2 apresenta a associação dos escores da qualidade de vida com características sociodemográficas dos docentes. Houve associação estatisticamente significativa $(p=0,037)$ entre o domínio físico e a idade, com maiores escores, 59,5 (DP $\pm 9,1$ ), para os com 44 anos ou mais, ou seja, nesse domínio, os mais velhos possuem qualidade de vida melhor que os mais jovens.

Em relação ao estado civil, houve associações significativas nos domínios psicológico $(p=0,009)$, relações sociais ( $p$ $=0,041)$ e meio ambiente, $(p=0,034)$, com maiores escores 


\begin{tabular}{|c|c|c|c|c|c|c|}
\hline \multicolumn{7}{|c|}{$\begin{array}{l}\text { Média e desvio-padrão dos escores dos domínios de qualidade de vida (Whoqol/breve) por categoria } \\
\text { profissional de docentes em uma universidade pública, Montes Claros (MG), Brasil 2014 (n = 221) }\end{array}$} \\
\hline & & & Média e desvio & & & \\
\hline Domínios & Biologia & Educação Física & Odontologia & Medicina & Enfermagem & $\begin{array}{c}\text { Média } \\
\text { geral }\end{array}$ \\
\hline Físico & $55,1+8,0$ & $58,5+9,0$ & $60,3+10,9$ & $60,0+9,6$ & $57,8+8,8$ & $57,9+9,6$ \\
\hline Psicológico & $64,8+1,3$ & $69,7+9,3$ & $66,6+11,7$ & $65,6+0,6$ & $66,5+11,5$ & $66,6+1,1$ \\
\hline Relações sociais & $71,3+2,8$ & $78,8+5,0$ & $78,0+16,9$ & $68,4+8,1$ & $72,5+18,9$ & $73,7+17,2$ \\
\hline Meio ambiente & $65,0+0,9$ & $73,0+4,7$ & $73,9+15,7$ & $68,3+14,5$ & $71,0+11,4$ & $70,4+13,9$ \\
\hline
\end{tabular}

\begin{tabular}{|c|c|c|c|c|c|}
\hline \multicolumn{6}{|c|}{$\begin{array}{l}\text { TABELA } 2 \\
\text { Escores médios nos domínios da qualidade de vida de acordo com as características sociodemográficas } \\
\text { dos docentes em uma universidade pública. Montes Claros (MG), Brasil. } 2014 \text { (n=221) }\end{array}$} \\
\hline \multicolumn{6}{|c|}{ Escores médios da qualidade de vida } \\
\hline Variáveis & n $(\%)$ & Físico & Psicológico & Relações sociais & Meio ambiente \\
\hline \multicolumn{6}{|l|}{ Sociodemográficas } \\
\hline \multicolumn{6}{|l|}{ Sexo } \\
\hline Feminino & $12857,9)$ & 57,7 & 65,9 & 72,4 & 70,6 \\
\hline Masculino & $93(42,1)$ & 58,2 & 67,7 & 75,7 & 70,2 \\
\hline $\mathrm{p}$ - valor (teste-t) & & 0,74 & 0,23 & 0,17 & 0,81 \\
\hline \multicolumn{6}{|l|}{ Idade } \\
\hline Até 43 anos & $130(58,8)$ & 56,8 & 65,8 & 74,3 & 69,5 \\
\hline 44 ou mais & $91(41,2)$ & 59,5 & 67,9 & 72,7 & 71,9 \\
\hline $\mathrm{p}$ - valor (teste-t) & & 0,03 & 0,16 & 0,48 & 0,21 \\
\hline \multicolumn{6}{|l|}{ Estado civil } \\
\hline Com companheiro & $15971,9)$ & 58,5 & 67,9 & 75,1 & 71,7 \\
\hline Sem companheiro & $62(28,1)$ & 56,3 & 63,5 & 69,8 & 63,3 \\
\hline $\mathrm{p}$ - valor (teste-t) & & 0,13 & 0,00 & 0,04 & 0,03 \\
\hline \multicolumn{6}{|l|}{ Cor autodeclarada } \\
\hline Branca & $125(56,6)$ & 58,2 & 66,4 & 74,7 & 71 \\
\hline Parda e outras & $96(43,4)$ & 57,5 & 67 & 72,4 & 69,7 \\
\hline $\mathrm{p}$ - valor (teste-t) & & 0,58 & 0,68 & 0,33 & 0,48 \\
\hline \multicolumn{6}{|l|}{ Onde reside } \\
\hline Em Montes Claros & $217(98,2)$ & 57,9 & 66,6 & 73,7 & 70,5 \\
\hline Outra cidade & $4(1,8)$ & 59,4 & 64,6 & 72,9 & 67,9 \\
\hline $\mathrm{p}$ - valor (teste-t) & & 0,76 & 0,71 & 0,92 & 0,71 \\
\hline \multicolumn{6}{|l|}{ Com quem mora } \\
\hline Com a própria família & $188(85,1)$ & 58 & 67,1 & 74,6 & 70,9 \\
\hline Outra situação & $33(14,9)$ & 57,6 & 63,8 & 68,2 & 67,6 \\
\hline $\mathrm{p}$ - valor (teste-t) & & 0,82 & 0,10 & 0,04 & 0,20 \\
\hline \multicolumn{6}{|l|}{ Lazer } \\
\hline Atividades sociais & $89(40,3)$ & 58,14 & 68,1 & 76,6 & 72,7 \\
\hline Atividades culturais & $132(59,7)$ & 57,8 & 65,6 & 71,7 & 68,9 \\
\hline $\mathrm{p}-$ valor (teste-t) & & 0,77 & 0,10 & 0,03 & 0,04 \\
\hline \multicolumn{6}{|l|}{ Renda bruta mensal } \\
\hline Acima de 11 salários & $130(58,8)$ & 58,2 & 67,5 & 73,8 & 72,9 \\
\hline Até 11 salários & $91(41,2)$ & 57,7 & 66,0 & 73,6 & 68,7 \\
\hline $\mathrm{p}$ - valor (teste-t) & & 0,72 & 0,33 & 0,92 & 0,02 \\
\hline
\end{tabular}




\begin{tabular}{|c|c|c|c|c|c|}
\hline \multicolumn{6}{|c|}{$\begin{array}{l}\text { Escores médios nos domínios da qualidade de vida de acordo com as características acadêmicas } \\
\text { dos docentes em uma universidade pública. Montes Claros (MG), Brasil. } 2014 \text { (n = 221) }\end{array}$} \\
\hline \multicolumn{6}{|c|}{ Escores médios da qualidade de vida } \\
\hline Variáveis & n $(\%)$ & Físico & Psicológico & Relações sociais & Meio ambiente \\
\hline \multicolumn{6}{|l|}{ Acadêmicas } \\
\hline \multicolumn{6}{|l|}{ Curso } \\
\hline Biologia & $34(15,4)$ & 55,1 & 64,8 & 71,3 & 65,0 \\
\hline Educação Física & $35(15,8)$ & 58,7 & 69,7 & 78,8 & 73,9 \\
\hline Odontologia & $50(22,6)$ & 60,3 & 66,6 & 78,0 & 73,0 \\
\hline Medicina & $48(21,7)$ & 56,9 & 65,6 & 68,4 & 68,3 \\
\hline Enfermagem & $54(24,4)$ & 57,8 & 66,5 & 72,5 & 71,0 \\
\hline p - valor (Anova) & & 0,15 & 0,39 & 0,01 & 0,03 \\
\hline \multicolumn{6}{|l|}{ Outro vínculo empregatício } \\
\hline Não & $80(36,2)$ & 58,01 & 67,5 & 76,35 & 71,2 \\
\hline Sim & $141(63,8)$ & 57,9 & 66,1 & 72,2 & 70,0 \\
\hline $\mathrm{p}$ - valor (teste-t) & & 0,90 & 0,35 & 0,08 & 0,53 \\
\hline \multicolumn{6}{|l|}{ Tempo de atuação na docência } \\
\hline Até 12 anos & $134(60,6)$ & 59,6 & 68,7 & 75,1 & 72,8 \\
\hline Acima de 12 anos & $87(39,4)$ & 56,8 & 65,3 & 72,8 & 68,9 \\
\hline $\mathrm{p}$ - valor (teste-t) & & 0,03 & 0,02 & 0,32 & 0,04 \\
\hline \multicolumn{6}{|c|}{ Nível máximo de formação universitária } \\
\hline Com pós stricto sensu & $130(58,8)$ & 57,8 & 66,6 & 74,2 & 12,7 \\
\hline Sem pós stricto sensu & $82(37,1)$ & 58,1 & 66,7 & 72,8 & 70,7 \\
\hline $\mathrm{p}$ - valor (teste-t) & & 0,80 & 0,96 & 0,54 & 0,84 \\
\hline \multicolumn{6}{|c|}{ Livros, excetuados os acadêmicos } \\
\hline Até 2 livros & $92(41,6)$ & 59,6 & 68,2 & 74,6 & 71,6 \\
\hline Acima de 2 livros & $179(58,4)$ & 55,6 & 64,4 & 72,4 & 68,9 \\
\hline $\mathrm{p}-$ valor (teste-t) & & 0,00 & 0,01 & 0,34 & 0,15 \\
\hline
\end{tabular}

médios para os que possuem companheiro, isto é, estes apresentam melhor qualidade de vida. O fato de morar com a própria família determinou maior escore médio, 74,6 ( $\mathrm{DP} \pm 14,2)$, para o domínio relações sociais $(\mathrm{p}=0,04)$. Em relação ao lazer, houve associação estatisticamente significativa nos domínios relações sociais $(p=0,039)$ e meio ambiente $(p=0,045)$, com maiores escores, respectivamente $76,6(\mathrm{DP} \pm 16,2)$ e 72,7 (DP $\pm 13,4$ ), para aqueles que dedicam mais tempo às atividades sociais, indicando melhor qualidade de vida para esses indivíduos, se comparados aos que dedicam mais tempo às atividades culturais. A maior renda esteve associada a maiores escores de qualidade de vida, 72,9 (DP $\pm 13,2$ ), no domínio meio ambiente $(p=0,029)$.

Como demonstrado na Tabela 3, em relação ao tipo de curso em que o docente atua, verificaram-se diferenças estatisticamente significantes nos domínios relações sociais e meio ambiente. Maiores escores médios da qualidade de vida foram observados para os docentes de Educação Física, 78,8 (DP \pm
5,0), e menores para os de Medicina, 68,4 (DP $\pm 8,1$ ), no domínio relações sociais $(\mathrm{p}=0,010)$. No domínio meio ambiente ( $\mathrm{p}$ $=0,030)$, os professores do curso de Educação Física obtiveram escores superiores, 73,9 (DP $\pm 4,7)$, enquanto no curso de Biologia houve menores escores, 65,0 (DP $\pm 0,9$ ). Quanto ao tempo de atuação na docência, ocorreu associação estatisticamente significativa nos domínios físico, psicológico e ambiente, com maiores escores para os docentes com até 12 anos de atuação na docência. Assim, professores com menos tempo de docência apresentam maior qualidade de vida.

Também houve associação estatisticamente significativa com a quantidade de livros lidos anualmente, excetuados os livros acadêmicos, com os domínios físico $(p=0,002)$ e psicológico ( $\mathrm{p}=0,013)$, com maiores escores, 59,6 (DP \pm 9,3) no físico e $68,2(\mathrm{DP} \pm 11,1)$ no psicológico, para os que leem até dois livros anualmente.

Quanto às demais características - sexo, cor autodeclarada, onde reside, outro vínculo empregatício, nível máximo de 
formação universitária, fumante, fonte de informação - não foram encontradas associações significativas.

\section{DISCUSSÃO}

O perfil sociodemográfico dos docentes participantes deste estudo foi semelhante ao encontrado em outras pesquisas sobre essa temática ${ }^{11,14,15}$.

Em relação à qualidade de vida, esta pesquisa mostrou qualidade de vida boa no domínio psicológico, relações sociais e meio ambiente, porém o domínio físico se apresentou como regular. Estudo similar, realizado em instituição de ensino superior no Sul do Brasil, utilizando o Whoqol-Breve, com o objetivo de avaliar a percepção de qualidade de vida e saúde e fatores de risco de 293 docentes, também verificou que houve maior escore para os domínios psicológico, relações sociais e meio ambiente, com valores semelhantes aos encontrados neste estudo ${ }^{16}$.

Investigação feita entre 517 professores de instituições públicas e privadas na cidade de Campina Grande (PB) para avaliar os índices de sentido de vida, bem-estar psicológico e qualidade de vida em uma amostra de educadores mostrou resultados diferentes dos encontrados neste estudo, uma vez que os domínios psicológico e social apresentaram qualidade de vida regular. Esse fato mostra a necessidade de olhar atentamente para a saúde dos docentes desse cenário ${ }^{15}$. Em contrapartida, o presente estudo obteve resultado bom para os domínios psicológico, relações sociais e meio ambiente.

Estudo similar a este, realizado com 203 docentes no Rio Grande do Sul com a finalidade de investigar a qualidade de vida de docentes de instituições de ensino superior comunitárias, identificou a média de 71,3 no domínio relações sociais ${ }^{13}$, semelhante à encontrada nesta investigação, ou seja, a qualidade de vida, assim como no presente estudo, também foi boa entre os docentes do Rio Grande do Sul.

Um estudo realizado na Universidade do Vale do Itajaí (Univali), em Santa Catarina, ao avaliar a qualidade de vida dos docentes, verificou que o domínio meio ambiente obteve o segundo menor escore ${ }^{17}$, diferentemente dos dados encontrados nesta pesquisa.

Na associação dos domínios com as variáveis sociodemográficas, no presente estudo, a idade obteve maior escore médio no domínio físico para os docentes com idade superior a 44 anos. Dados semelhantes foram encontrados entre os 203 professores investigados de universidades do Rio Grande do Sul, em que os docentes com idade superior a 40 anos tiveram melhor qualidade de vida ${ }^{13}$. Entretanto, vale salientar que o domínio físico inclui aspectos como dor e desconforto, sono e repouso, atividades da vida cotidiana e capacidade de traba- lho. Tais aspectos tendem a ser comprometidos pelo avançar da idade, afetando negativamente a qualidade de vida, como revelado em estudo elaborado em outra instituição pública também do Sul do Brasil, onde as percepções mais negativas de saúde aumentaram com o decorrer da idade ${ }^{16}$.

A variável estado civil, nesta pesquisa, obteve dados significativos nos domínios psicológico, relações sociais e meio ambiente, com maiores escores para os docentes que possuem companheiro. Estudo semelhante, realizado no Rio Grande do Sul com o objetivo de analisar os parâmetros individuais e socioambientais da qualidade de vida percebida na carreira, com 380 educadores, mostrou que professores casados obtiveram boa qualidade de vida ${ }^{18}$. Uma pesquisa conduzida em Natal (RN) também mostrou dados similares ao desta pesquisa, em que os docentes casados possuem melhor qualidade de vida ${ }^{14}$.

Observa-se que professores casados e, supostamente, com rotina familiar já estabelecida podem adotar comportamentos mais saudáveis em comparação aos colegas de mesma profissão que não têm companheiro. Apesar disso, estes últimos não precisam se preocupar em demasia em satisfazer desejos, expectativas e anseios de um companheiro formal e/ou de seu(s) dependente(s) ${ }^{19}$. Todavia, essa realidade pode ser distinta para homens e mulheres, mas no presente estudo não se identificou diferença significativa entre os sexos.

Outro estudo, que teve como objetivo analisar a percepção de qualidade de vida e saúde e a incidência de fatores de riscos modificáveis em professores de uma instituição pública de ensino superior do Sul do Brasil observou que os professores universitários, com bom nível econômico, possuem melhor qualidade de vida no domínio meio ambiente ${ }^{16}$. Na presente pesquisa, a renda bruta mensal acima de 11 salários mínimos favoreceu o maior escore no domínio meio ambiente, o que confirma semelhanças entre os achados. Nessa perspectiva, a questão salarial e a valorização profissional são fatores que podem proporcionar melhores condições de vida e melhor qualidade de vida ${ }^{11}$. Quando se investiga a valorização da função docente no âmbito acadêmico, tanto na percepção do imaginário social quanto nos planos de cargos, carreiras e salários, verifica-se que o docente do ensino superior público encontra-se em boa posição. Por isso, o resultado foi positivo para a maioria dos participantes desta pesquisa no aspecto de remuneração e estabilidade alcançadas por meio do vínculo atual de trabalho ${ }^{20}$.

A associação dos domínios com as variáveis acadêmicas, nesta pesquisa, mostrou que os educadores que exercem a carreira docente há menos de 12 anos alcançaram maiores escores nos domínios físico, psicológico e meio ambiente. Um estudo semelhante, ao avaliar a qualidade de vida de 73 discentes por 
meio do Whoqol-Breve em Maceió (AL), verificou que o menor tempo de carreira docente esteve associado a maiores escores de qualidade de vida no domínio físico, de forma semelhante ao encontrado na presente pesquisa ${ }^{21}$. A conjectura para esses achados se mostra mais favorável aos profissionais com menor tempo de carreira, os quais podem auferir melhores condições de saúde e maior disposição para as atividades de trabalho. Por outro lado, é pertinente refletir que os docentes atuantes há mais tempo e que têm mais idade geralmente sentem maior segurança, estabilidade emocional e profissional, lidam melhor com as adversidades, compreendem melhor o posicionamento de colegas, acadêmicos e chefia, além de suas expectativas serem outras ${ }^{13}$. Embora esse contexto colabore positivamente para a sua qualidade de vida, na presente investigação, os docentes mais velhos apresentaram escores inferiores nos citados domínios da qualidade de vida.

Identificou-se, ainda, que quem mora com a família apresenta boa qualidade de vida. No entanto, uma investigação realizada com 349 docentes em Florianópolis (SC), também pelo Whoqol-Breve, com o objetivo de investigar a percepção de qualidade de vida dos docentes, verificou menor escore de qualidade de vida para as pessoas que moram com a família ${ }^{22}$. Mesmo considerando que a dupla rotina dos docentes pode acarretar problemas familiares e de saúde, entende-se que a família é de grande importância para o desenvolvimento de seus membros. É necessária a participação de todos para que possam desempenhar satisfatoriamente suas funções ${ }^{23}$.

A prática profissional docente é marcada por fatores e sentimentos que comprometem a qualidade de vida em geral. A avaliação da qualidade de vida é um primeiro passo para se refletir sobre esses aspectos e compartilhar as situações que a influenciam positiva ou negativamente. A partir do momento em que se possibilita ao docente perceber situações que afetam sua qualidade de vida, isso pode proporcionar-lhe o início da busca por melhores condições de vida e de trabalho ${ }^{17}$.

Os professores do curso de Educação Física obtiveram maiores escores nos domínios relações sociais e meio ambiente, ao passo que os professores de Medicina e Biologia obtiveram escores inferiores. Esse achado vai ao encontro de investigação sobre a experiência do trabalho docente em cursos de saúde de uma universidade federal da Região Norte do Brasil. Embora o exercício profissional tenha sido identificado como fonte de estabilidade e realização pessoal e financeira, destacaram-se a precariedade do trabalho e relações interpessoais desgastadas, que tendem a induzir sofrimento e adoecimento $^{20}$. Isso pode ter afetado de forma mais acentuada os docentes dos cursos de Biologia e Medicina do presente estudo. Assim, é necessária uma reflexão sobre a temática entre docentes, instituições de ensino e sociedade, na perspectiva de promover melhor qualidade de vida para esse público ${ }^{8,20}$.

\section{CONCLUSÃO}

A boa qualidade de vida encontrada na maioria dos domínios pode oferecer momentos positivos aos docentes da área da saúde da instituição deste cenário. Todavia, como os professores que participaram deste estudo obtiveram qualidade de vida regular no domínio físico, esse aspecto demanda atenção, uma vez que a associação de uma boa qualidade de vida em todos os domínios favorece melhor qualidade de vida de maneira geral. A associação das características sociodemográficas e acadêmicas com a qualidade de vida mostrou a necessidade de prestar maior cuidado aos docentes que exercem a carreira há mais de 12 anos, não apresentam companheiro, não residem com a família e apresentam idade inferior a 43 anos, evidenciando maior necessidade de intervenção para esses trabalhadores. Ressalta-se a demanda de atenção especial aos docentes dos cursos de Biologia e Medicina, que apresentaram qualidade de vida mais afetada.

Embora este estudo seja restrito a uma única instituição de ensino superior e a descrição da qualidade de vida tenha sido feita de maneira abrangente, é relevante investigar essa temática, na perspectiva de propiciar melhoria na vida diária e formação dos docentes. Assim, recomenda-se a realização de outras pesquisas no cenário da presente investigação e em outros locais de ensino. Espera-se que este estudo subsidie as instituições de ensino superior com ações efetivas no campo da promoção da saúde e no fomento de avanços na qualidade de vida acadêmica.

\section{AGRADECIMENTOS}

À Unimontes, pela bolsa do Programa Institucional de Bolsas de Iniciação Científica (Pibic).

\section{REFERÊNCIAS}

1. Minayo MCS, Hartz ZMA, BUSS PM. Qualidade de vida e saúde: um debate necessário. Ciênc Saúde Colet. 2000;5(1):7-18.

2. Seidl EMF, Zannon CMLC. Qualidade de vida e saúde: aspectos conceituais e metodológicos. Cad Saúde Pública. 2004;20(2):580-8.

3. Petrini AC. Avaliação da percepção da qualidade de vida de jovens universitários: comparativo entre graduandos do turno diurno e noturno. Rev Bras Qualidade de Vida. 2013;5(3):1-8.

4. Arronqui GV, Lacava RMVB, Magalhães SMF, Goldman RE. Perceptions of nursing students on their quality of life. Acta Paul Enferm. 2011;24(6):762-5. 
5. Almeida BAM, Guitierrez LG, Marques R. Qualidade de vida: definição, conceitos e interfaces com outras áreas de pesquisa. São Paulo: Escola de Artes, Ciências e Humanidades, 2012.

6. Koifman L. A função da universidade e a formação médica. Rev Bras Educ Med. 2011;35(2):145-6.

7. Garcia AL, Oliveira ERA, Barros EB. Qualidade de vida de professores do ensino superior na área da saúde: discurso e prática cotidiana. Cogitare Enferm. 2008;13(1):18-24.

8. Ferreira RC, Silveira AP, Barbosa de Sá MA, Feres SBL, Souza JGS, Martins AMEBL. Transtorno mental e estressores no trabalho entre professores universitários da área da saúde. Trab Educ Saúde. 2015;13(supl. 1):135-55.

9. Martinez KASC, Vitta A, Lopes ES. Avaliação da qualidade de vida dos professores universitários da cidade de Bauru-SP. Salusvita. 2009;28(3):217-24.

10. Ramos FRS, Borges LM, Brehmer LCD, Silveira LR. Ethical education of nurses - indicators of change on the perception of professors. Acta Paul de Enferm. 2011;24(4):485-92.

11. Dávila MHX, Casagrande RJT, Pereira VCG. Qualidade de vida do trabalhador de uma instituição de ensino. Cad Esc Saúde. 2010;1(4):110-26.

12. Alves JGB, Tenório M, Anjos AG, Figueroa JN. Qualidade de vida em estudantes de Medicina no início e final do curso: avaliação pelo Whoqol-bref. Rev Bras Educ Med. 2010;34(1):91-6.

13. Koetz L, Rempel C, Périco E. Qualidade de vida de professores de instituições de ensino superior comunitárias do Rio Grande do Sul. Ciênc Saúde Colet. 2013;18(4):1019-28.

14. Fernandes MH, Rocha VM, Fagundes AAR. Impacto da sintomatologia osteomuscular na qualidade de vida de professores. Rev Bras Epidemiol. 2011;14(2):276-84.

15. Damásio BF, Melo RLP, Silva JP. Sentido de vida, bem-estar psicológico e qualidade de vida em professores escolares. Paidéia. 2013;23(54):73-82.

16. Filho AO, Netto-Oliveira ER, Oliveira AAB. Qualidade de vida e fatores de risco de professores universitários. Rev Educ Fís/UEM. 2012;23(1):57-67.

17. Cogo LLR, Gonçalves LO, Kerkoski E, Santos AA, Chesani FH. Perfil da qualidade de vida dos fisioterapeutas docentes do curso de fisioterapia da Universidade do Vale do Itajaí. Rev Contexto Saúde. 2011;10(20):367-74.
18. Lemos CAF, Nascimento JV, Borgatto AF. Parâmetros individuais e sócio-ambientais da qualidade de vida percebida na carreira docente em Educação Física. Rev Bras Educ Fís Esporte. 2007;21(2):81-93.

19. Salles WN, Egerland EM, Barroso MLC, Souza CA. Estilo de vida e perfil socioeconômico de docentes dos cursos de Educação Física da Universidade Federal de Santa Catarina - UFSC. Rev Bras Ciênc Saúde. 2012;10(34):7-14.

20. Lago RR, Cunha BS, Borges MFSO. Percepção do trabalho docente em uma universidade da região norte do Brasil. Trab Educ Saúde. 2015;13(2):429-50.

21. Morais EPG, Azevedo RR, Chiari BM. Correlação entre voz, autoavaliação vocal e qualidade de vida em voz de professoras. Rev CEFAC. 2012;5(14):892-900.

22. Pereira EF, Teixeira CS, Lopes ADS. Qualidade de vida de professores de educação básica do município de Florianópolis, SC, Brasil. Ciênc Saúde Colet. 2013;18(7):1963-70.

23. Silva MLS, Arpini DM. A nova lei nacional de adoção - desafios para a reinserção familiar. Psicol Estud. 2013;18(1):125-35.

\section{CONTRIBUIÇÃO DOS AUTORES}

Souto LES, Souza SM, Lima CA e Lacerda MKS participaram de todas as etapas da pesquisa: concepção e desenho do estudo, coleta de dados, análise e interpretação dos dados, elaboração e revisão final do artigo. Vieira MA orientou todas as etapas da pesquisa, participou da concepção e desenho do estudo, interpretação dos dados, elaboração e revisão final do artigo. Costa FM e Caldeira AP participaram da concepção e delineamento do estudo, análise e interpretação dos dados, revisão crítica do artigo e revisão da versão final.

\section{CONFLITO DE INTERESSES}

Os autores declaram não haver conflito de interesses.

\section{ENDEREÇO PARA CORRESPONDÊNCIA}

Lyssa Esteves Souza Souto

Rua Joaquina da Glória, 56

Panorama II - Montes Claros

CEP 39401-603 - MG

E-mail: lyssaesteves@yahoo.com.br 